\section{Dye-DNA-Lipid Thin Films Exhibit Durable Amplified Spontaneous Emission}

A considerable obstacle in the current development of solid-state lasers is durability. Researchers at the Chitose Institute of Science and Technology, Japan, have demonstrated that thin films composed of DNA, lipid, and a hemicyanine dye have the potential for a practical, durable, solid-state laser.

As reported in the August 19 issue of Applied Physics Letters, Y. Kawabe and coworkers fabricated thin films by casting on Teflon plates a dichloromethane-methanol solution containing DNA-hexadecyltrimethylammonium (HTMA) and 4-[4(dimethyamino) stylyl]-1-dococylpyridinium bromide (DMASDPB), a derivatized hemicyanine dye known for its nonlinear optical properties. Self-standing films were formed by evaporating the solvent in vacuum. Film thickness was controlled by varying the concentration. Fluorescence enhancement of DMASDPB in DNA was previously confirmed.

Threshold-energy levels were determined by measuring the light intensity emitted from the samples as a function of pump-pulse energy. The output of a frequency-doubled $\mathrm{Nd}^{3+}$ :YAG laser $(\lambda=$ $\left.532 \mathrm{~nm}, \tau_{\mathrm{p}}=7 \mathrm{~ns}\right)$, used as the pump source, was focused with a normal incident angle onto a $1 \mathrm{~mm} \times 5 \mathrm{~mm}$ stripe on the sample. The emitted light was observed perpendicular to the incident beam along the direction of the stripe.

Emission peaked at $631 \mathrm{~nm}$ under lowintensity pumping. A shift in the emission peak to $624 \mathrm{~nm}$ with concomitant spectral narrowing were observed under highintensity pumping. While output energy is proportional to pump energy in the low-intensity region, superlinear dependence is evident for energies greater than $0.5 \mathrm{~mJ} / \mathrm{cm}^{2}$. The researchers attribute the spectral narrowing to amplification because the narrowing and superlinear enhancement occur at the same intensity. Amplification occurs at relatively low selfabsorption. In addition, the emitted light is linearly polarized parallel to the film surface, indicating enhanced amplification. The researchers conclude from these results that amplified spontaneous emission occurs in the DMASDPB-HTMA films.

The role played by DNA was investigated in two control experiments. First, thin films were fabricated with poly(methyl methacrylate) and DMASDPB. The resulting films showed strong fluorescence under the same pumping conditions, but neither intensity enhancement nor spectral narrowing was observed. In the second control experiment, a simple laser cavity containing a chloroform solution of DMASDPB was prepared. No indication of lasing was observed. The same apparatus confirmed lasing for some conventional laser dyes as well as for a chloroform solution of DMASDPB and HTMA. The researchers believe that this clearly demonstrates that DMASDPB acquires the ability to lase through its interaction with DNA, which likely alters the dye's electronic state. Further structural and spectroscopic studies to investigate the details of the dye-DNA interaction are planned by the researchers.

In this preliminary study, which was performed in air at room temperature, a reduction in lasing performance of only a few percent was observed after two hours of operation; the total fluence of energy during the test was $210 \mathrm{~J} / \mathrm{cm}^{2}$. The researchers believe that the low threshold, good performance, and long durability exhibited by their DMASDPBHTMA films demonstrate their potential for practical use as solid-state lasers, especially because their method can be applied to chromophores other than conventional laser dyes.

\section{STEVEN TROHALAKI}

\section{DNA-Templated Nanowire}

\section{Fabrication Technique Developed}

As the feature sizes of electronic circuits approach the limits of optical lithography, the costs of traditional micro- and nanolithography techniques used in the electronics industry will become unacceptably high. Recently, several groups have studied approaches using DNA for nanowiring. However, due to the relatively poor intrinsic electric conductivity of DNA, metallization is necessary. In search of new fabrication technologies, a research group from Sony International (Europe) $\mathrm{GmbH}$ reported in the September issue of Nano Letters on their technique for metallizing DNA using negatively charged tris(hydroxymethyl)phosphine-capped gold nanoparticles (THP-AuNPs).

An aqueous colloidal sol of THP-AuNPs was synthesized by mixing $\mathrm{HAuCl}_{4}$ solution and hydrolyzed tetrakis(hydroxymethyl)phosphonium chloride solution. The resultant particles were characterized by lithium-ion exchange, inductively coupled plasma-atomic emission spectroscopy analysis, and gel electrophoresis. The results showed that the particles were negatively charged with an average individual net charge of approximately $15 e$.

Two steps are involved in metallization: formation of conjugates between DNA and metal nanoparticles (NPs) and enlargement of the NPs by electroless plating. In the first step, in situ or ex situ

\section{Find what \\ you need in 5 minutes or less}

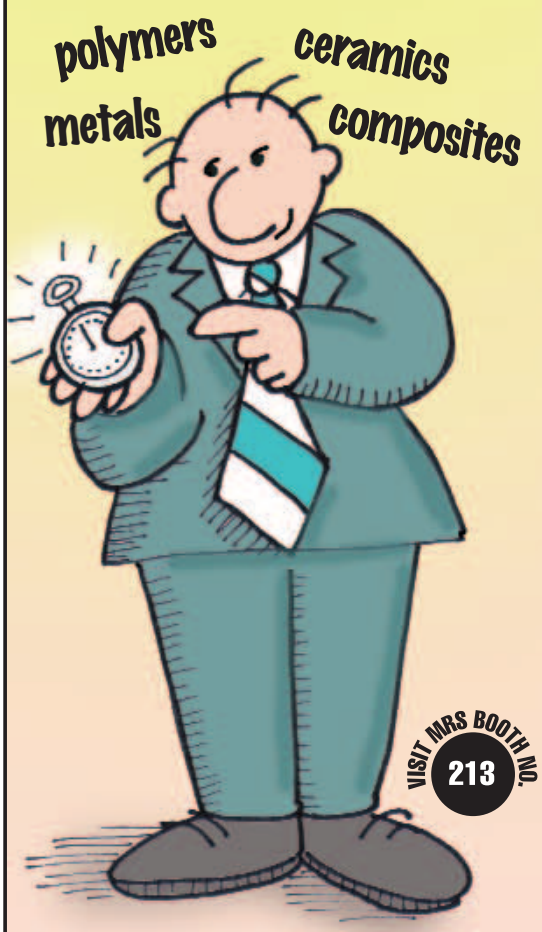

You'll find more than 40,000 different items in our online Catalog, the majority in stock and ready for shipment.

Need something special?

We can help with that, too. Take a moment now and see how Goodfellow can help.

Web: www.goodfellow.com (Secure, online ordering)

Real live person: 1-800-821-2870 E-mail: info@goodfellow.com Fax: 1-800-283-2020

\section{FIOIFEl|III METALS \& MATERIALS FOR RESEARCH \& INDUSTRY}

(c) 2002 Goodfellow Corporation

Circle No. 14 on Inside Back Cover 
approaches are used, based on the formation of the metal NPs in the DNA-NP conjugate. In this research, the ex situ method was employed because the researchers discovered that negatively charged THPAuNPs can bind densely to the negatively charged calf thymus DNA. During the metallization procedure, DNA was first immobilized on a silicon substrate by $\mathrm{O}_{2}$ plasma treatment to enhance immobilization and spin-coating to elongate the molecules. Before the THP-AuNP sol was applied to the substrate, the particles were precipitated in ethanol, then dissolved in an ethanol-water solution. Optimum results with respect to density of particle templating, avoidance of excess particles on the substrate, and lack of defects in the resultant wires were obtained at an ethanol:water ratio of 95:5. After rinsing and drying, the samples were treated with an electroless gold plating solution in order to form conductive nanowires for further characterization.

Atomic force microscopy revealed that after calf thymus DNA molecules on a silicon substrate were treated with THP-AuNPs in $95 \%$ ethanol, the DNA molecules were decorated with particles, with some excess particles ( $0.7 \mathrm{~nm}$ to $2 \mathrm{~nm}$ in size) also on the substrate. Scanning electron microscopy images showed that the electroless gold plating of the resulting DNAAuNP conjugates provides nanowires $\sim 30-40 \mathrm{~nm}$ in width and longer than $2 \mu \mathrm{m}$. The good contrast against the silicon surface indicates high conductivity and the metallization was restricted almost entirely to the DNA. The results of the electrical transport capabilities of the DNA nanowires showed that their electrical conductivities were about one-thousandth that of bulk gold.

To determine what kind of mechanism controls the binding between THP-AuNPs and DNA, several hypotheses were considered, based on the interactions between THP-AuNPs and DNA, such as adsorption of THP-AuNPs to hydrophilic DNA molecules on the silicon oxide surface, hydrogen-bonding interactions in between, ligand replacement by groups on DNA that can coordinate to the AuNP surface, and the covalent bonding between THP and amino groups of the DNA base.

The process can be finished in $\sim 10 \mathrm{~min}$, but the in situ approaches that employ metal salts or complexes as precursors take longer than $1 \mathrm{~h}$. The technique reported here by $\mathrm{O}$. Harnack and coworkers could be used for future electronic circuits because it can lower the fabrication cost and is suitable for feature sizes below the current limit of optical lithography. However, issues about the binding mechanism between the
THP-AuNPs and DNA, and how the solvent and substrate influence the process, need to be further studied.

YUE HU

\section{Direct Ink-Jet Printing Assembles $\mathrm{ZrO}_{2}$ Powder into 3D Shape}

Three-dimensional shapes can be created from ceramic powders by forcing droplets of "ink" containing the powder through an ink-jet nozzle, layering the deposition to generate height. Xinglong Zhao and colleagues from the University of London in conjunction with Jin-Hua Song from Brunel University report their work on the creation of vertical walls from ceramic materials using ink-jet printing in the August issue of the Journal of the American Ceramic Society. Zhao and colleagues have created mazes (replicas of one at Hampton Court Palace outside London) with various wall-thickness values where the smallest gap between walls was $170 \pm 10 \mu \mathrm{m}$. This technique has applications in rapid prototyping and the creation of ceramic molds, circuit boards, and biosensors.

$\mathrm{A} \mathrm{ZrO}_{2}$ powder with an average particle size of $0.45 \mu \mathrm{m}$ was mixed with a commercial dispersant to create a mixture with an estimated $\mathrm{ZrO}_{2}$ powder volume fraction of 0.63 . The combination was then stirred with an ultrasonic probe for $5 \mathrm{~min}$ and milled for approximately $30 \mathrm{~min}$ to disperse the powder into the liquid and break down the agglomerates. The ink-jet print head contained $50050-\mu \mathrm{m}$ nozzles with a print width of $70 \mathrm{~mm}$ and a computer-controlled sliding table. The table print speed was $500 \mathrm{~mm} / \mathrm{s}$ and registered its position at the edge of the track between passes to ensure accuracy in the $y$ printing direction. To assure uniformity,

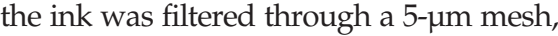
and the air was removed from the system by repeated extraction and insertion of the ink through the print head until no signs

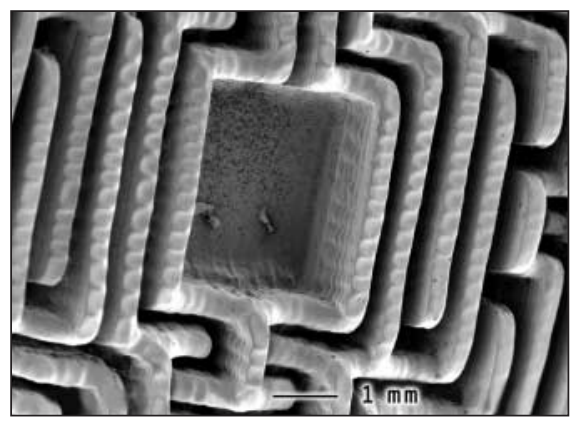

Figure. Scanning electron micrograph of vertical ceramic walls created by direct ceramic ink-jet printing. of bubbles appeared in the tubing. Between passes, a hot-air blower was used for $20 \mathrm{~s}$ to enhance the drying procedure and reduce spreading. Since the ink remained opaque after $10 \mathrm{~h}$, the final samples were allowed to dry for $24 \mathrm{~h}$ before pyrolyzation and sintering.

The results of this process based on a maze pattern can be seen in the figure. Although individual droplets cannot be seen, an overall surface texture results in part from coalescence during the drying process. Very straight vertical walls were achieved, although the height was not uniform due to a wall-thickness effect, where thinner walls had reduced height, and also because of in-filling of the center parts of the maze. The researchers said that complications resulting from ink drying and spreading will need to be considered during the scaling-up process.

CHRISTINE RUSSELL

\section{Simulations Show a Hexatic Phase in Porous Media}

In the Kosterlitz-Thouless-HalperinNelson-Young (KTHNY) theory, when a two-dimensional crystal melts, it first becomes hexatic by the unbinding of dislocation pairs, and then becomes liquid through the unbinding of disclination pairs. However, early simulations did not exhibit the hexatic phase. Now, researchers at the Massachusetts Institute of Technology, North Carolina State University, and Adam Mickiewicz University in Poland have observed the hexatic phase, consistent with the theory, in molecular simulations of simple fluids in narrow slitshaped carbon pores. The stability of the phase increased with the strength of the fluid-wall interaction. Experimental measurements confirmed the existence of the hexatic phase.

In Monte Carlo simulations of large systems of the order of 64,000 molecules, the crystalline-hexatic and hexatic-liquid transitions were observed for simple fluids of spherical molecules. R. Radhakrishnan of MIT and co-workers reported in the August 12 issue of Physical Review Letters that they modeled the fluid-fluid interaction of $\mathrm{CCl}_{4}$ with a Lennard-Jones potential and the fluid-wall interaction with a 10-4-3 Steele potential.

The simulated pores were of two different widths, $0.911 \mathrm{~nm}$ or $1.41 \mathrm{~nm}$, and contained either one or two layers of adsorbed $\mathrm{CCl}_{4}$. The researchers monitored the in-plane positional and orientational correlation functions to determine the nature of the confined phase.

For the two-layer system, at $360 \mathrm{~K}$ the positional correlation function was isotropic, and the orientational one showed 